Thélème. Revista Complutense de Estudios Franceses

ISSN-e: 1989-8193

http://dx.doi.org/10.5209/THEL.55339

\title{
Transposer l'étrangeté : la traduction de poètes francophones des Antilles
}

\author{
Clara Curell ${ }^{1}$
}

Recibido: 24 de febrero de 2017 / Aceptado: 18 de mayo de 2017

Résumé. Les difficultés spécifiques que pose la traduction de la variation linguistique et, en particulier, celle des textes littéraires écrits dans des variétés topolectales riches en connotations et en références à des réalités culturelles particulières ont suscité l'intérêt de nombreux spécialistes, notamment à partir des années quatre-vingt-dix lorsque s'est produit ce qui a été appelé le tournant culturel de la traductologie. Dans cet article, nous allons illustrer à l'aide d'exemples appartenant à l'œuvre poétique de différents auteurs franco-antillais contemporains (Guy Tirolien, Ernest Pépin, Joseph Zobel et Nicole Cage-Florentiny) certains des problèmes traductologiques qui se posent ainsi que les stratégies qui peuvent être mises en place pour traduire en espagnol les signifiés dénotatifs et connotatifs de lexèmes diatopiquement marqués en préservant au maximum leur altérité.

Mots clés : traduction poétique; variation topolectale; français des Antilles; poètes francophones des Caraïbes.

\section{[es] Trasladar la alteridad: la traducción de poetas franco-antillanos}

Resumen. Las dificultades específicas que suscita la traducción de la variación lingüística y, en especial, la de textos literarios escritos en variedades topolectales cargadas de connotaciones y de referencias a realidades culturales específicas han despertado el interés de numerosos estudiosos, principalmente a partir de los años noventa cuando se produjo el denominado giro cultural de la traductología. En este artículo nos proponemos ilustrar con ejemplos pertenecientes a la obra poética de varios autores franco-antillanos contemporáneos (Guy Tirolien, Ernest Pépin, Joseph Zobel y Nicole Cage-Florentiny) algunos de los problemas traductológicos que se plantean, así como las estrategias que se pueden adoptar con el fin de trasladar al español los significados denotativos y connotativos de lexemas diatópicamente marcados preservando al máximo su alteridad.

Palabras clave: traducción poética; variación topolectal; francés antillano; poetas del Caribe francófono.

\section{[en] Rendering Otherness: the Translation of Francophone Caribbean Poets}

Abstract. Since the 1990s and the growing influence of the so-called 'cultural turn' in Translation Studies, considerable research has been devoted to the difficulties arising from the translation of linguistic variation, particularly the translation of literary texts written in linguistic varieties with culture-specific references. Drawing on the poetic works of a several contemporary Francophone Caribbean authors (Guy Tirolien, Ernest Pépin, Joseph Zobel and Nicole Cage-Florentiny) we analyze in this paper some of these translation issues and suggest strategies that can be adopted so as to render in Spanish both

Universidad de La Laguna

ccurell@ull.edu.es 
denotative and connotative meanings of the lexical items specific to the variety, and at once preserving their local character.

Keywords: poetic translation; topolectal variation; Caribbean French; Francophone Caribbean poets.

Cómo citar: Curell, C. (2017). «Transposer l'étrangeté : la traduction de poètes francophones des Antilles ». Thélème. Revista Complutense de Estudios Franceses, Vol. 32, Núm. 2 : 159-173

L'essence de la traduction est d'être ouverture, dialogue, métissage, décentrement.

Elle est mise en rapport ou elle n'est rien.

(Antoine Berman, L'épreuve de l'étranger, 1984)

La complexité et les difficultés spécifiques que pose la traduction de la variation linguistique et, en particulier, celles de textes littéraires écrits dans des variétés diatopiquement marquées, riches en éléments transculturels et où abondent les désignations d'une réalité propre (realia ou culturèmes) ont été soulignées par un bon nombre de spécialistes de différents domaines linguistiques, notamment à partir des années quatre-vingt-dix lorsque s'est produit ce qu' on a appelé le tournant culturel de la traductologie ${ }^{2}$. En effet, comme l'a signalé Vidal Claramonte (2007 : 62), les nouvelles approches culturalistes (telles que la traduction féministe, la traduction postcoloniale ou la théorie de la manipulation et de la réécriture) ont mis en exergue - outre le contexte socio-politique, qui est toujours présent dans l'acte de traduire - le rôle du traducteur, non tant comme un simple passeur du sens des mots que comme un médiateur interculturel. Comme tel, le traducteur doit essayer d'identifier, de transposer et d'expliquer l'altérité au-delà des mots en choisissant soigneusement le lexique, en appliquant la stratégie traductive la plus adéquate et en prenant souvent position sur des questions éthiques, esthétiques et idéologiques. Si nous considérons ainsi la traduction comme un acte de décision qui implique, à différents niveaux, le choix de certaines options au détriment d'autres, la soi-disant neutralité ou invisibilité du traducteur s'évanouit :

Comme le processus traductologique est profondément ancré dans la culture, il n'est jamais dénué d'idéologie. Explicitement ou implicitement, les mots véhiculent des sens colorés et connotés. Le choix des mots et du style révèle ou dissimule, il est dans tous les cas rarement neutre (Guillaume, $2016: 17$ ).

C'est ainsi que face au texte étranger et, en particulier, en présence de discours culturellement marqués, nous nous trouvons confrontés à deux conceptions antagonistes de la traduction, qui s'inscrivent dans le débat classique entre sourciers et ciblistes, termes formulés par Ladmiral en $1986^{3}$ pour distinguer, d'une part, les

\footnotetext{
Nous citons ci-dessous quelques monographies parmi les nombreuses qui abordent cette question dans les domaines espagnol et français : Palimpsestes, 6 (1991) ; Carbonell i Cortés (1994) ; Palimpsestes, 11 (1998); Carbonell i Cortés (1999); Palimpsestes, 12 (2000); Ballard (2005) ; Wecksteen et El Kaladi (2007) ; Ortega Arjonilla (2007); Lombez et von Kulessa (2007) ; Camps et Zybatow (2008); Palimpsestes, 25 (2012).

3 Ladmiral reprend ainsi la célèbre opposition conceptuelle exposée en 1955 par Georges Mounin, issue des théories de Friedrich Schleiermacher, entre « les verres transparents », qui font référence aux traductions qui semblent avoir été rédigées dans la langue cible, et « les verres colorés », qui désignent ces autres traductions conservant une série d'éléments du texte original qui rappellent constamment au lecteur qu'il se trouve en présence d'une œuvre traduite.
} 
traducteurs qui prônent la préservation de l'équivalence formelle de l'original et, d'autre part, ceux qui privilégient la réexpression du signifié dans le texte cible. Cette dichotomie a été relancée plus tard par Venuti (1995 et 1998), un des traductologues qui a le plus insisté sur la visibilité du traducteur, afin d'opposer l'approche exotisante ou foreignization, qui cherche à conserver les traits linguistiques et culturels du texte source et qu'il préconise, à une stratégie traductive vernaculaire ou domestication, qui tend à occulter la différence et à uniformiser le texte étranger. Suivant la visée d'Antoine Berman (1999 : 69 et ss.) de respecter l'altérité comme une éthique de la traduction, Venuti (1995 : 14 et ss.) estime ainsi que le traducteur doit mettre en relief cette diversité et provoquer un effet de défamiliarisation en conservant dans sa version certains repères du discours étranger tels que l'organisation textuelle de la langue source, l'imitation de ses structures syntactiques ou même la reproduction ou le calque de mots originaux.

Ceci nous amène directement au cas concret de la traductibilité de discours écrits dans une variante vernaculaire, pour lesquels certains spécialistes appartenant au domaine hispanique (notamment Julià Ballbé, 1997 ; Hurtado Albir, 2001 et Marco Borillo, 2002), tout en reconnaissant la difficulté de trouver une véritable correspondance, coïncident avec Venuti dans son aspiration à restituer, de façon partielle ou totale, les traits dialectaux. Cela étant, lorsque la modalité diatopique est la langue de narration de l'ouvrage entier, Berman (1999 : 64) lui-même prévient que « le vernaculaire ne peut être traduit dans un autre vernaculaire. Une telle exotisation, qui rend l'étranger du dehors par celui du dedans, n'aboutit qu'à ridiculiser 1'original ». Ceci explique que nous partageons l'opinion de Carbonell i Cortés (2004 : 32-47) de faire appel, dans ces cas, à une méthode de traduction éclectique entre ces deux extrêmes, en respectant l'idiosyncrasie du texte étranger, mais sans l'exagérer de façon artificielle, en veillant non tant à reproduire la variété topolectale qu'à rendre les évocations et les connotations qu'elle véhicule. D'autre part, comme nous l'avons déjà indiqué plus haut, ces textes contiennent d'habitude une quantité non négligeable de diatopismes qui renvoient à des réalités propres, très souvent sans équivalent dans la langue cible. Pour aborder leur traduction, outre le fait évident de disposer de la compétence linguistique nécessaire, le traducteur doit posséder une connaissance approfondie de la culture étrangère qu'il doit essayer de conserver compensant la tendance quasi inévitable à produire une traduction neutralisante à l'aide d'une série de démarches visant à combler les éventuelles lacunes aussi bien linguistiques que culturelles de la traduction. En plus des stratégies que Ballard (2003: 149-156 et $2005: 130-135)$ propose pour inscrire l'altérité, c'est-à-dire le report pur et simple (normalement marqué typographiquement, ce qui amène le lecteur à chercher le sens de ce mot), le report assorti d'une note explicative, l'incrémentialisation ou explicitation entre parenthèses ou entre virgules et la note, il existe d'autres procédés péritextuels tels que les préfaces et les postfaces ou encore l'ajout d'un bref lexique de diatopismes en fin de volume. Toutefois, certains de ces paratextes comme les notes en bas de page ou les glossaires doivent être utilisés avec beaucoup de prudence dans la traduction de textes littéraires, car ils détournent le regard du lecteur et interrompent fâcheusement la lecture. Si nous nous en tenons au cas singulier de la traduction de poésie, qui est celui que nous allons analyser dans ce travail, nous estimons que les notes du traducteur doivent être réduites au minimum et, le cas échéant, avoir un caractère explicatif visant simplement à combler un écart lexiculturel, sauf si - cela va de soi - il s'agit 
d'une édition annotée. Une autre option parmi les formules envisagées par Ballard, la glose intratextuelle, est tout aussi inapplicable dans la traduction de poésie où il faut tenir compte, outre la dimension référentielle, du dispositif formel du poème et de certaines contraintes rythmiques et métriques qu'il faut transposer de la manière la plus équivalente possible. Nous sommes d'avis qu'une façon de contrecarrer l'impossibilité de recourir à cette stratégie clarificatrice est de présenter les poèmes en version bilingue reproduisant l'original en regard de sa traduction, ce qui permet une circulation entre les deux textes et aide le lecteur à comprendre ou à deviner certaines des décisions prises par le traducteur.

Dans cet article, nous aborderons par conséquent les problèmes spécifiques et les limites que pose la translation de textes diatopiquement marqués et regorgeant de références culturelles, en utilisant un échantillon de mots - en grande mesure des dénominations de realia - que nous estimons illustratifs et que nous avons extraits de recueils de poèmes de quatre auteurs franco-antillais contemporains : Joseph Zobel $^{4}$, Guy Tirolien ${ }^{5}$ Ernest Pépin ${ }^{6}$ et Nicole Cage-Florentiny ${ }^{7}$. Ces représentants de la francophonie antillaise, à l'instar de nombreux autres écrivains de la région, utilisent une variété linguistique que certains érudits appellent un « interlecte » entre le français et le créole (cf. Prudent, $1981: 26$ ). Ainsi, du point de vue lexical, c'est une modalité qui se caractérise, d'une part, par la persistance d'archaïsmes et de régionalismes appartenant à la langue des colons français du XVII siècle et, d'autre part, par la présence d'apports du créole se rapportant au monde caraïbe, d'adaptations de mots espagnols, anglais, amérindiens et africains, et de néologismes internes qui évoquent également des réalités propres. Sur le plan morpho-syntaxique, il s'agit d'un topolecte qui présente certaines caractéristiques de la langue parlée ainsi que de nombreuses influences du créole : confusion de genre, de nombre, de personne, de temps et de modes verbaux sans oublier l'addition ou l'omission de déterminants, de pronoms, de conjonctions ou de prépositions.

C'est pour cette raison, et comme étape préalable à la traduction, que notre première tâche a été de repérer et d'identifier une sélection de lexies, vérifiant leur éven-

Le Martiniquais Joseph Zobel (1915-2006), auteur d'une dizaine de romans et de nouvelles en plus de plusieurs recueils de poèmes, est surtout connu pour La rue Cases-Nègres (1950), un ouvrage qui fait partie du cursus scolaire martiniquais et qui a été porté à l'écran avec grand succès en 1982. Pour cette étude, nous nous sommes limités à sa production poétique, publiée dans le livre Le soleil m'a dit... (2012).

5 Le Guadeloupéen Guy Tirolien (1917-1988) fait partie, depuis ses débuts, du mouvement littéraire de la Négritude, avec Léopold Sédar Senghor, Aimé Césaire ou Léon-Gontran Damas. Il est l'auteur de quelques nouvelles et de plusieurs recueils de poèmes, notamment Balles d'or (1961), qui contient sa célèbre « Prière d'un petit enfant nègre » (1943) et d'autres compositions que nous avons analysées pour cette occasion.

6 Également guadeloupéen, Ernest Pépin (né en 1950) fait preuve d'un profond engagement envers la culture caribéenne, l'antillanité et le créolisme tout en revendiquant, comme ses contemporains Jean Bernabé, Raphaël Confiant ou Patrick Chamoiseau, une « identité-mosaïque » ouverte sur le monde. Son œuvre littéraire se limite essentiellement à la poésie et au récit. Pour documenter cette étude, nous avons analysé deux de ses recueils de poèmes : Boucan de mots libres (éd. bilingue français-espagnol 1991 ; éd. française 2005) et Solo d'îles (2010).

7 Nicole Cage-Florentiny (Martinique, 1965) est journaliste, professeur d'espagnol, chanteuse et, par-dessus tout, écrivaine. Son engagement envers sa région ainsi que le pouvoir thérapeutique qu'elle attribue au mot expliquent qu'elle utilise souvent le créole pour donner libre cours à sa veine créatrice. Son anthologie en français Arc-en-ciel, l'espoir (1996) nous a donné quelques exemples pour notre corpus. 
tuel caractère diatopique, pour effectuer ensuite leur décodage complet en utilisant les ouvrages lexicographiques et bibliographiques appropriés. C'est ainsi que nous avons consulté, en plus des ressources dictionnairiques habituelles pour la traduction vers l'espagnol, des dictionnaires différentiels du français antillais (Telchid, 2007 ; BDLP) et des lexiques créole-français ou encore créole-anglais (Confiant, 2007 ; Valdman, 2007), ainsi que certaines études linguistiques récentes sur cette variété diatopique (Thibault, 2008a, 2008b, 2010 et 2014 ; Zanoaga, 2012). Quand nous avons pu disposer de versions espagnoles des poèmes en question, notre analyse nous a permis de comparer - et, le cas échéant, de corriger ou de nuancer - les solutions présentées. Lorsqu'aucune traduction préalable n'était disponible, nous avons proposé notre propre option visant à la fois la recherche de la correspondance sémantique et la préservation, dans la mesure du possible, de la richesse de la langue source et des valeurs culturelles qui lui sont associées ${ }^{8}$.

Le premier terme que nous analysons est le substantif féminin avalasse, auquel le TLF consacre une simple vedette de renvoi à avalaison, sous lequel il est répertorié sans aucun marquage diasystémique avec le sens de «torrent formé par de grosses pluies ou par la fonte subite des neiges ». De son côté, le Robert donne à l'entrée avalaison ou avalasse une définition pratiquement identique : "cours d'eau torrentiel qui descend soudainement des montagnes à la suite de pluies abondantes ou de fontes des neiges " , tandis que les deux dernières éditions du Dictionnaire de l'Académie française $\left(8^{\mathrm{e}}\right.$ et $\left.9^{\mathrm{e}}\right)$ ne lui accordent aucune entrée, même pas un renvoi. La BDLP nous propose une signification différente de celle du français de référence, à savoir celle de " pluie torrentielle, grosse averse, déluge », relevée en Louisiane, et ajoute que ce mot est également usité aux Seychelles et à La Réunion dans le sens de « inondation violente consécutive à des pluies torrentielles ». En ce qui concerne son étymologie, cette même source signale qu'il s'agit d'un maintien d'un lexème de l'ancien français et des parlers régionaux de France, bien attesté dans les parlers de l'Ouest (même s'il ne semble pas être en usage de nos jours dans le français régional, étant donné qu'il n'est pas cité dans le DRF). Bien que Telchid ne l'inclue pas, ce terme figure cependant chez Valdman comme un régionalisme d'Haïti qui possède son corrélat créole lavalas et qui désigne un « déluge », ce qui est confirmé chez Confiant où il est défini comme " pluie torrentielle ». En effet, dans les Antilles françaises, on appelle avalasses les pluies fréquentes, concentrées surtout en fin d'après-midi, qui se déclenchent pendant l'hivernage.

Dans les textes analysés, le mot apparaît à deux reprises dans un même poème de Nicole Cage-Florentiny (1996), intitulé « Fils d'espérance », qui a été traduit par la poète cubaine Nancy Morejón.

On constatera que dans certains cas nous avons quelque peu délaissé les aspects métriques et rythmiques, ce qui signifie que, du point de vue de la traduction poétique, nos propositions sont de simples versions provisoires qui s'inscrivent dans une phase intermédiaire du processus de traduction. 
Cage-Florentiny, « Fils d'espérance »

(v. 23 et v. 29)

Une pluie avalasse

$[\ldots]$

Et la pluie avalasse fera tout le reste !
Traduction de Nancy Morejón

Una avalancha de lluvia

$[\ldots]$

Y la avalancha de lluvia hará el resto

La traductrice l'a correctement identifié comme étant un diatopisme sémantique, attendu qu'elle ne l'a pas interprété dans le sens qui figure dans les dictionnaires du français standard, mais elle n'a pas remarqué que pluie avalasse est une composition faite à partir de termes quasi redondants (hyperonyme-hyponyme), calquée sur le créole lapli-lavalas. De ce fait, elle a produit une version littérale, mot à mot, probablement guidée par la similitude phonétique entre avalasse et avalancha, alors que nous estimons qu'elle aurait pu traduire ces deux termes au contenu récurrent par une lexie simple comme chaparrón ou, mieux encore, aguacero, plus courant en espagnol méridional et qui reprend, dans une certaine mesure, la marque topolectale. Quoi qu'il en soit, si l'on accordait la priorité aux contraintes métriques, une possibilité de traduction neutre pourrait être le syntagme nominal lluvia impetuosa.

Le deuxième exemple de notre échantillon est le nom féminin coulée, vocable qui a le sens de « petite vallée étroite » (Telchid) et qui provient des dialectes occidentaux de France (BDLP). Il est bien attesté actuellement sous ce sens, non seulement dans la littérature antillaise, mais aussi dans des textes de différentes variétés du français nord-américain (Québec, Acadie et Louisiane). Il est également répertorié avec la graphie kulé dans des sources créoles (Thibault, 2008a : 117). En ce qui concerne le français général, et comme l'attestent les principaux dictionnaires consultés (TLF, Littré, DCLF), le mot désigne, en parlant d'un liquide, d'une masse pâteuse ou en fusion : « le fait de s'écouler; ce qui s'écoule », " flot de lave, etc. ».

Dans notre corpus, nous trouvons le mot dans deux compositions d'Ernest Pépin (1991), « Paroles de mon pays » et « Méditations », traduites par Nancy Morejón, ainsi que dans le poème « Ode » de Joseph Zobel (2002).

Dans le premier des deux contextes, nous estimons que la traductrice n'a pas saisi la condition de régionalisme sémantique du substantif et l'a interprété, à tort, dans son sens standard, alors que la présence d'un autre diatopisme concernant la géographie antillaise (morne) aurait dû dissiper, à notre avis, le moindre doute. Ainsi, si nous donnons priorité au sens et sacrifions la coloration régionale, son équivalent en espagnol neutre serait l'hyperonyme valle, même si l'on pourrait également utiliser les mots barranco, quebrada ou cañón, qui partagent avec le mot original le sème /étroit/.

Pépin, « Paroles de mon pays » (v. 10-14)

Paroles de mon pays

de mornes en mornes

au cœur des coulées

en laitage d'arbre-à-pain

en cillements de feuilles-à-bain
Traduction de Nancy Morejón

Palabras de mi país

de cerro en cerro

en el corazón de los metales fundidos

en el lacticinio del árbol del pan

en el parpadear de las hojas para bañarse 
Par contre, dans le deuxième exemple, Pépin utilise le mot plutôt dans son acception générale, mais la proposition de la traductrice, metales fundidos, outre le fait d'être excessivement prosaïque, ne nous semble pas non plus juste. Nous estimons qu'il serait possible d'envisager ici d'autres options plus appropriées, comme río ou reguero.

$\begin{array}{ll}\text { Pépin, « Méditations » (v. 1-6) } & \text { Traduction de Nancy Morejón } \\ \begin{array}{l}\text { Du fond des profondeurs } \\ \text { lit du monde surgi }\end{array} & \begin{array}{l}\text { Del fondo de las profundidades } \\ \text { lecho del mundo vuelto a surgir }\end{array} \\ \text { frutailles aux riches ornements du désir } & \begin{array}{l}\text { frutillas de ricos ornamentos del deseo } \\ \text { haut sur les colonnes du temple }\end{array} \\ \begin{array}{l}\text { la rive désertique } \\ \text { et la révélation d'une coulée d'astres claires columnas del templo }\end{array} & \begin{array}{l}\text { y la revelación de vidrio fundido de astros } \\ \text { claros }\end{array}\end{array}$

Dans les vers de Zobel, le terme coulée est également utilisé dans le sens du français de référence, raison pour laquelle nous proposons de le rendre par flujo dans la traduction métrique sous forme d'endécasyllabes et d'heptasyllabes qui suit :

\author{
Zobel, « Ode » (v. 87-91) \\ et cette résonance dans la voix \\ où se fondent les harmoniques \\ de peine et de joie \\ répandus sur le cœur \\ en une coulée fertilisante
}

\author{
Notre traduction \\ y estas resonancias en la voz \\ que funden los armónicos \\ de pena y de alegría \\ vertidos en el pecho \\ en un flujo fecundo
}

D'après André Thibault (2010 : 50), un des experts qui a le plus approfondi l'étude de la francophonie linguistique ces dernières années, le nom morne est l'un des vocables emblématiques du français antillais (attesté depuis 1640, TLF), qui s'est étendu par la suite aux langues vernaculaires de La Réunion et du Canada. Il est utilisé, en particulier en parlant d'une île ou d'un littoral, pour désigner une « colline, monticule, petite montagne » (Telchid) et, bien que son origine soit incertaine, on indique qu'il pourrait provenir de l'espagnol morro (TLF, DCLF), malgré certains problèmes phonétiques inexplicables (cf. Thibault, 2014: 280).

Or, il est notoire que morne est un terme polysémique : en effet, en français de référence, c'est un adjectif qui s'applique à une personne « qui est dans un état d'abattement, d'ennui ou de tristesse » ou à une chose « qui provoque un état de lassitude, d'ennui par son uniformité, son absence d'intérêt, son manque d'éclat " (TLF). Dans notre sélection, nous avons trouvé plusieurs exemples (Cage-Florentiny, 1996 ; Tirolien, 1961 ; Pépin, 1991 et 2010) dans lesquels ce mot est utilisé, sans équivoque, dans l'acception qu'il possède aux Antilles, même si, dans les versions que nous avons consultées (Morejón, 1996 ; Cortés, 2006 ; Morejón, 1991 et Oliver, 2010), il est généralement traduit par des équivalents sémantiques en espagnol neutre. Une suggestion de traduction qui pourrait peut-être récupérer, dans une certaine mesure, la nature régionale de morne consisterait à recourir précisément à son étymon probable, morro, " monte pequeño o peñasco redondeado » (DLE), qui est 
habituel dans certaines variétés de l'espagnol méridional et qui fait partie de nombreux toponymes aussi bien dans la péninsule ibérique et les Iles Canaries que dans des régions sud-américaines. Une autre possibilité, pour autant que les contraintes métriques le permettent, serait d'utiliser le synonyme loma, fréquent dans le parler de Cuba. D'autre part, comme on peut le constater ci-dessous, l'erreur de traduction commise par Armando González-Pérez et Barry D. Amis est pour le moins étonnante : ils n'ont pas reconnu le régionalisme, qu'ils ont confondu avec l'adjectif morne substantivé, et l'ont traduit par le nom féminin espagnol tristeza.

Cage-Florentiny, « Fils d'espérance » (v. 2024)

Ma mère écoute. Elle dit :

Je refuse la déveine...

Moi, j'ai rêvé la paix

Une pluie avalasse

Dégringolant des mornes nus

Tirolien, « Prière d'un petit enfant nègre » (v. 1-6)

[Seigneur

Je suis très fatigué

Je suis né fatigué

Et j'ai beaucoup marché depuis le chant du coq]

et le morne est bien haut

qui mène à leur école

Pépin, « Paroles de mon pays » (v. 10-14)

Paroles de mon pays

de mornes en mornes

au cœur des coulées

en laitage d'arbre-à-pain

Pépin, « Solo d'îles » (v. 193-197)

Nous avons choisi de renaître

De ressusciter la tête des mornes

De nommer les plantes

De baptiser les bêtes

De faire chanter les arbres
Traduction de Nancy Morejón

Mi madre escucha. Dice:

Rechazo el maleficio...

Yo soñé con la paz

Una avalancha de lluvia

Precipitándose desde los cerros desnudos
Traduction de Rosalía Cortés

y es muy alta la colina

que lleva hasta su escuela

Traduction d'A. González-Pérez et B. D. Amis

y la tristeza que conduce a su escuela está marcada

\author{
Traduction de Nancy Morejón \\ Palabras de mi país \\ de cerro en cerro \\ en el corazón de los metales fundidos \\ en el lacticinio del árbol del pan
}

Traduction de José M. Oliver

Hemos elegido renacer

Resucitar la cima de los cerros

Dar un nombre a las plantas

Bautizar a los animales

Hacer cantar a los árboles

Le quatrième cas que nous présentons est la lexie composée pou de bois (var. poulbois), bien attestée dans les dictionnaires et encyclopédies des XVII ${ }^{\mathrm{e}}$ et XVIII ${ }^{\mathrm{e}}$ siècles, qui correspondrait littéralement à l'espagnol piojo de la madera. Elle possède un corrélat créole, poul-bwa (Confiant), et est habituellement utilisée dans le français antillais au lieu du terme non marqué termite (DCLF ; Telchid ; Zanoaga, 2012 : 444). Il s'agit donc d'un régionalisme de fréquence qui, d'autre part, n'a pas 
d'équivalent en espagnol, raison pour laquelle sa couleur locale ne peut pas être rendue en langue cible, comme on peut le constater dans l'exemple d'Ernest Pépin (2010) ci-dessous. Comme nous l'avons déjà signalé (Curell et Oliver, 2015 : 164), dans la culture populaire guadeloupéenne, et notamment lors des fêtes de Carnaval, il n'est pas rare de rencontrer des femmes portant un mas a poulbwa (ou poudbwa), « masque de poux de bois », c'est-à-dire, déguisées en femme termite?

Pépin, « Solo d’îles » (v. 128-131)

Solo d'îles

En résistance d'orage

En résistance de femmes poux de bois

En résistance de femme
Traduction de José M. Oliver

Solo de islas

Con fuerza de tormenta

Con fuerza de mujer termita

Con fuerza de mujer

Nous en venons maintenant au substantif quimbois et à ses dérivés (verbe quimboiser, noms quimboiseur, -euse) qui reviennent souvent dans les textes franco-antillais, au même titre que leurs corrélats créoles (kenbwa, kyenbwa, tjienbwa..., cf. BDLP et Zanoaga, 2012 : 455). Il a le sens de « sortilège, maléfice » (Telchid) et équivaut aux pratiques magico-religieuses syncrétiques d'origine africaine que l'on retrouve dans d'autres zones des Caraïbes et d'Amérique (vaudou, santería, candomblé, umbanda, etc.). Son origine est obscure, bien qu'on envisage comme étymon possible l'expression «tiens, bois ! », phrase dite par le sorcier lorsqu'il administre un philtre (Jourdain apud Thibault, 2008 : 297).

Le seul exemple que nous avons trouvé dans les textes sélectionnés (Zobel, 2002) apparaît sous la forme du nom féminin quimboiseuse, " personne qui jette des maléfices » (BDLP) :

$\begin{array}{ll}\text { Zobel, « Malavoi » (v. 23-25) } & \text { Notre traduction } \\ \text { Les quimboiseuses } & \text { Y las santeras } \\ \text { y perdent } & \text { pierden } \\ \text { leur boule de cristal } & \text { su bola de cristal }\end{array}$

Notre proposition exclut les termes non marqués bruja ou hechicera au profit de santera, substantif qui est utilisé de façon habituelle dans certaines modalités de l'espagnol d'Amérique (Cuba, Venezuela) et ne néglige pas le caractère diatopique du vocable franco-antillais et les connotations culturelles qui lui sont associées.

Dans la littérature française des Antilles, la forme féminine ravine, dont la distance sémantique avec ravin a été neutralisée depuis longtemps, possède une fréquence d'usage beaucoup plus élevée que ce dernier terme, les deux vocables désignant une « petite vallée étroite traversée par un cours d'eau » (BDLP). Par contre, le français de référence actuel connaît une différence de sens entre les deux car ravine

\footnotetext{
Les costumes traditionnels de Guadeloupe sont un héritage des masques africains. Durant la période du Carnaval, les esclaves avaient le droit de se déguiser et ils faisaient semblant de participer à la fête pour pratiquer leurs cérémonies initiatiques et d'exorcisme (cf. « Voukoum » in Potomitan. Site de promotion des cultures et des langues créoles. Document en ligne : http://www.potomitan. info/gwadloup/voukoum.php).
} 
distingue en particulier « un petit ravin » ou « le lit d'un torrent, d'un ruisseau », l'acception « torrent » étant vieillie (TLF, DCLF). De là que Thibault (2010: 50 et 2014 : 282) considère le nom ravine comme un antillanisme de fréquence.

Les versions espagnoles de ravine dont nous disposons (Tirolien, 1961 ; Pépin, 1991) se contentent d'équivalents tels que torrente et desfiladero qui sacrifient la coloration régionale. Nous estimons, en outre, que cette dernière proposition est assez éloignée du sens du mot original. C'est pourquoi nous pensons que l'on pourrait envisager d'autres possibilités de traduction plus réussies, comme barranco, barranca ou quebrada, mots habituels en espagnol d'Amérique et qui reproduiraient dans une certaine mesure la marque géolectale. Dans notre traduction du poème « Îles » de Guy Tirolien, nous avons opté précisément pour le terme quebrada.

Tirolien, « Prière d'un petit enfant nègre » $(\mathrm{v}$. 9-11)

Je veux suivre mon père dans les ravines fraîches

[Quand la nuit flotte encore dans le mystère des bois

Où glissent les esprits que l'aube vient chasser]

Pépin, « Je t'emmènerai en poésie » (v. 4-8)

Je t'emmènerai en poésie

Là où le ventre n'est plus qu'un paysage aux mains tendres

du matin

le désir aux ravines chante son oraison

Pépin, «Amants » (v. 6-9)

à grands tours de hanche

de paroles-ravines festoyant roucoulement d'orages

cueilli à plein ciel

Tirolien, « Îles » (v. 12-13)

Souffrance d'un long hennissement grimpant la pente des ravines
Traduction de Rosalía Cortés

Quiero ir tras de mi padre por los torrentes frescos

Traduction d'A. González-Pérez et B. D. Amis

Quiero seguir a mi padre por los frescos desfiladeros

\author{
Traduction de Nancy Morejón \\ Te llevaré conmigo en la poesía \\ Allá en donde el vientre no es más que un \\ paisaje \\ en las manos tiernas \\ de la mañana \\ el deseo torrencial canta su oración
}

\author{
Traduction de Nancy Morejón \\ a fuerza de caderas \\ de palabras-torrente en su fiesta \\ ronroneo de huracanes \\ en pleno cielo
}

\author{
Notre traduction \\ Dolor de un relinchar intenso \\ que sube el talud de las quebradas
}

Le créolisme pipirit (var. pipirite, Telchid) est probablement un vocable d'origine onomatopéique (Rézeau, $2008: 220$ ) désignant un « oiseau de la famille des Tyrannidés dont le chant, très matinal, annonce l'aube » (Confiant). Son caractère matinal a donné lieu aux expressions caraïbes opipiri et opipiri-chantan, ainsi qu'à leurs 
versions correspondantes en français des Antilles au pipirit et au pipirit chantant, qui ont toutes deux le sens de « à l'aube » (Confiant).

Nicole Cage-Florentiny (1996) nous fournit, dans différents poèmes, deux exemples de cette lexie correspondant respectivement aux deux acceptions. Dans le premier cas, l'auteur utilise le mot composé oiseau-pipirit, construction à base de termes quasi redondants (hyperonyme français et hyponyme créole), très productive en français des Antilles.

\author{
Cage-Florentiny, « Viens mon enfant » (v. 1-2) \\ Je sais trouver les traces des sources \\ cachées \\ Et dénicher l'oiseau-pipirit au fin-fond des \\ bois
}

\author{
Traduction de Nancy Morejón \\ Yo sé encontrar las fuentes de las huellas \\ escondidas \\ Y sacar de su nido al pájaro cantor al fino \\ fondo de los bosques
}

La traduction que propose Nancy Morejón ne nous paraît pas satisfaisante, non seulement parce qu'elle n'identifie pas l'oiseau en question, mais également parce qu'elle semble ignorer ce syntagme lexicalisé et, par conséquent, le processus particulier de création dont il est issu. C'est ainsi que Morejón se limite à traduire l'hyperonyme en ajoutant de son propre cru une épithète, sans parler d'autres inexactitudes de sa version, cette question ne faisant pas l'objet de cette étude. À notre avis, une des solutions que l'on pourrait envisager est l'équivalent de pipirit en espagnol de Cuba et Puerto Rico, pitirre, un mot également onomatopéique qui ressemble au cri de cet oiseau (DLE). Une autre option serait l'utilisation d'un néologisme occasionnel, pájaro-pitirre, en calquant mot à mot la lexie composée d'origine.

Dans la seconde composition de la poète martiniquaise, nous observons que pipirit est employé dans son sens métonymique de " à l'aube ", "à l'aurore ", ce qui n'a pas été saisi par la traductrice et l'amène à faire une interprétation peu heureuse :

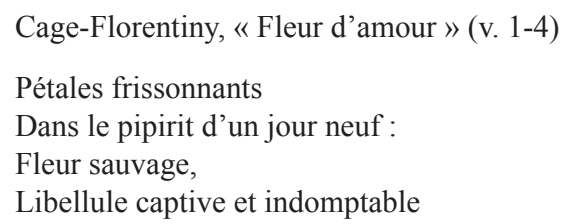

\author{
Traduction de Nancy Morejón \\ Pétalos trémulos \\ En el cantar de un nuevo día : \\ Flor silvestre, \\ Libélula cautiva e indomable
}

Pour terminer cet inventaire, nous avons choisi le mot case, qui évoque en français antillais n'importe quel type d'habitation et non un logis rudimentaire construit en matériaux légers, sens habituel en français métropolitain (Thibault et Drouin, 2010 : 293). Nous nous trouvons par conséquent en présence d'un diatopisme sémantique, c'est-à-dire, d'une forme qui existe en français standard et qui a pris un autre sens, dans ce cas hyperonymique, dans cette variété topolectale. Sa diffusion dans la région franco-antillaise a été favorisée, plus que probablement, par la coïncidence de l'espagnol casa, également utilisé dans les Antilles (TLF).

Ceci explique que, dans les trois cas repérés (Zobel, 2002; Tirolien, 1961), nous ayons préféré le rendre par l'hyperonyme espagnol casa, - ce qui nous a empêché de préserver l'étrangéité du terme d'origine - au lieu de recourir aux vocables $c a$ - 
baña ou choza qui correspondraient aux acceptions du français général « habitation rudimentaire... » (TLF) ou " petite et chétive maison » (Littré). Ci-dessous nous présentons nos propositions de traduction métrique :

\author{
Zobel, « Ballade » (v. 31-35) \\ Tous les sentiers viennent à moi \\ et m'entraînent \\ de case en case \\ au bord de l'eau \\ au fond des bois
}

Zobel, « Coco Charlot » (v. 190-195)

Le cœur comme une case sans enfants sans jardins ni soleil

Une case

sans chien ni chat ni fleurs

Tirolien, « Circoncision » (v. 4-6)

Le coryphée :

Serpent, ô serpent, pourquoi ce regard fixe?

Que cherches-tu dans la case des vierges?

\author{
Notre traduction \\ Todas las sendas llegan hacia mí \\ y me transportan \\ de casa en casa \\ cerca del agua \\ al fondo de los bosques \\ Notre traduction \\ El corazón lo mismo que una casa \\ sin niños \\ sin jardines \\ ni sol \\ Una casa \\ sin perro ni gato y sin flores
}

L'analyse de ce petit échantillon, qui nous semble suffisamment représentatif malgré son caractère limité, a permis de constater que, face à un texte écrit dans une variété géolectale, l'acte de traduction doit adopter une approche spécifique, surtout en ce qui concerne les realia, qui constituent un lexique sans équivalent dans la langue cible. C'est ainsi que, dans le souci de reproduire le réseau culturel inhérent à ce type de discours, le traducteur est censé se documenter de façon appropriée sur la réalité étrangère et s'assurer de saisir convenablement la signification des mots ou expressions topolectales, en les différenciant, le cas échéant, de leur sens et de leur usage dans la langue standard. Une documentation solide et le recours à des ressources lexicographiques différentielles adéquates - lorsqu'elles existent - peuvent contribuer à identifier ces variantes, à cerner leur registre et leur fréquence, à éviter l'utilisation de faux sémantismes et à se rapprocher autant que possible de l'équivalence recherchée. Lorsqu'il s'agit de transposer les régionalismes lexicaux, nous avons observé qu'il existe plusieurs stratégies traductologiques, mais les plus efficaces seront celles qui permettront de contrecarrer, dans une certaine mesure, l'opacité sémantique qui caractérise ces mots. Cela étant, comme nous avons pu le vérifier en analysant les propositions de différents traducteurs, il est plus difficile - quand ce n'est pas impossible - de reproduire les associations connotatives véhiculées par les formes diatopiquement marquées. Selon le genre textuel dont il s'agit, nous pouvons avoir recours à des reports et à des calques, et voire à la création de néologismes, ou 
encore insérer des paraphrases explicatives dans le texte lui-même. Il est également possible de compléter la traduction avec des énoncés paratextuels rendant visible une partie de ce qui se perd dans la traduction. Toutefois, dans le cas concret d'un corpus poétique comme celui qui est analysé dans cette étude, où certains de ces procédés ne sont pas applicables ou sont déconseillés, la compétence du traducteur est encore plus mise à l'épreuve, comme en témoignent les mots de Christine Raguet (2007: 41):

La tâche du traducteur ne peut s'assimiler à un acte standardisateur, aseptiseur qui a pour objet l'homogénéisation d'une source soi-disant unique s'inscrivant au cœur d'une cible aux limites bien définies sans tenir compte des mélodies, des harmonies de la ligne rythmique, dont les échos signifiants dépassent le bornage de la compréhension.

\section{Références bibliographiques}

Ballard, M., (2003) Versus : la version réfléchie. Repérages et paramètres. Paris, Ophrys.

Ballard, M., (2005) «Les stratégies de traduction des désignateurs de référents culturels », in Ballard, M. (éd.), La traduction, contact de langues et de cultures. Arras, Artois Presses Université, pp. 125-152.

BDLP = Agence universitaire de la Francophonie et Trésor de la Langue française au Québec, (2001-2014) Base de données lexicographiques panfrancophone [En ligne]. Disponible sur : http://www.tlfq.ulaval.ca/bdlp [Dernier accès le 20/02/2017].

Berman, A., (1984) L'épreuve de l'étranger. Paris, Gallimard.

Berman, A., (1999) La traduction et la lettre ou l'auberge du lointain. Paris, Seuil.

Cage-Florentiny, N. (1996) Arc-en-ciel, l'espoir / Arco iris, la esperanza. Édition bilingue. Traduction de Nancy Morejón. La Habana, Ediciones Casa de las Américas.

Camps, A. \& L. Zybatow (éds.), (2008) Traducción e interculturalidad. Actas de la Conferencia Internacional "Traducción e intercambio cultural en la época de la globalización», mayo de 2006, Universidad de Barcelona. Frankfurt am Main, Peter Lang.

Carbonell i Cortés, O., (1994) Traducir al Otro: traducción, poscolonialismo. Cuenca, Universidad de Castilla-La Mancha.

Carbonell i Cortés, O., (1999) Traducción y cultura. De la ideología al texto. Salamanca, Ediciones Colegio de España.

Carbonell i Cortés, O., (2004) « La ética del traductor y de la traductología », in Grupo TLS (éd.), Ética y política de la traducción literaria. Málaga, Miguel Gómez Ediciones, pp. 17-46.

Confiant, R., (2007) Dictionnaire créole martiniquais-français. Matoury, Ibis Rouge éditions.

Cortés, R. R., (2006) « Poesía del Caribe francófono. Haití y Antillas francesas », in Palimpsestes. No 5, pp. 184-196.

Curell, C. \& J. M. Oliver, (2015) « Entre lirismo y exotismo : el reto de traducir a Ernest Pépin », in Fumero Pérez, M C. \& J. J. Batista Rodríguez, (éds.), Cuestiones de lingüística teórica y aplicada. Frankfurt am Main, Peter Lang, pp. 161-170.

$\mathrm{DCLF}=\mathrm{A}$. Rey (dir.) (2005) Dictionnaire culturel de langue française. Paris, Dictionnaires Le Robert. 
Dictionnaire de l'Académie française. $8^{\mathrm{e}}$ édition. 1932-1935 [En ligne]. Disponible sur : http://atilf.atilf.fr/academie.htm [Dernier accès le 20/02/2017].

Dictionnaire de l'Académie française. $9^{\mathrm{e}}$ édition. En cours de rédaction [En ligne]. Disponible sur : http://atilf.atilf.fr/academie9.htm [Dernier accès le 20/02/2017].

DLE = Real Academia Española (2014): Diccionario de la lengua española. Madrid: Espasa Calpe, 23 édition. [En ligne]. Version informatisée disponible sur : http:// www.rae.es/diccionario-de-la-lengua-espanola/la-23a-edicion-2014 [Dernier accès le 20/02/2017].

$\mathrm{DRF}=$ P. Rézeau (éd.) (2001) Dictionnaire des régionalismes de France: géographie et histoire d'un patrimoine linguistique. Bruxelles, De Boeck / Duculot.

González-Pérez, A., (1976) Antología clave de la poesía afroamericana. Madrid, Ediciones Alcalá.

Guillaume, A., (dir.) (2016) Idéologie et traductologie. Paris, L'Harmattan.

Hurtado Albir, A., (2001) Traducción y traductología. Introducción a la traductología. Madrid, Cátedra.

Julià Ballbé, J., (1997) « Dialectes i traducció: reticències i aberracions », in Bacardí, M. (éd.), Actes del II Congrés Internacional sobre Traducció. Bellaterra, Servei de Publicacions de la Universitat Autònoma de Barcelona, pp. 561-574.

Ladmiral, J.-R., (1986) «Sourciers et ciblistes », in Revue d'esthétique. No 12, pp. 33-42.

Le Robert = A. Rey (dir.) (2001) Le Grand Robert de la langue française. Paris, Dictionnaires Le Robert.

Littré, É., (1863-1872) Dictionnaire de la langue française. Paris, Hachette.

Lombez, Ch. \& R. von Kulessa, (2007) De la traduction et des transferts culturels. Paris, L'Harmattan.

Marco Borillo, J., (2002) El fil d'Ariadna. Anàlisi estilística i traducció literària. Barcelona, Eumo.

Mounin, G., (1955) Les belles infidèles. Paris, Éditions des Cahiers du Sud.

Ortega Arjonilla, E. (éd.), (2007) El giro cultural de la traducción. Reflexiones teóricas y aplicaciones didácticas. Frankfurt am Main, Peter Lang.

Pépin, E., (1991) Boucan de mots libres / Remolino de palabras libres. Édition bilingue. Traduction de Nancy Morejón. La Habana, Ediciones Casa de las Américas.

Pépin, E., (2005) Boucan de mots libres. Nancy, Éditions Aspect.

Pépin, E., (2010) Solo d'îles. Odyssée de la ville / Solo de islas. Odisea de la ciudad / Um solo de ilhas. Odisseia da cidade. Traduction espagnole de José M. Oliver. Traduction portugaise d'Ana Isabel Moniz et Thierry Proença dos Santos. Islas Canarias, Gobierno de Canarias (coll. Horizontes Insulares, Septenio).

Prudent, L.-F., (1981) « Diglossie et interlecte », in Langages. $N^{\circ}$ 61, pp. 13-38.

Raguet, Ch., (2007) «Y a-t-il des limites à la traduction transculturelle ? », in Wecksteen C. \& A. El Kaladi (éds.), La traductologie dans tous ses états. Arras, Artois Presses Université, pp. 39-54.

Rézeau, P., (2008) «Aspects du français et du créole des Antilles (notamment Saint-Domingue) à la fin du XVIII ${ }^{\mathrm{e}}$ siècle, d'après le témoignage d'un lexicographe anonyme », in Thibault, A. (coord.), Richesses du français et géographie linguistique. Bruxelles, De Boeck / Duculot, vol. 2, pp. 195-226.

Telchid, S., (1997) Dictionnaire du français régional des Antilles : Guadeloupe, Martinique. Paris, Bonneton.

Thibault, A., (2008a) « Français des Antilles et français d'Amérique : les diatopismes de Joseph Zobel, auteur martiniquais », in Revue de Linguistique Romane. № 72, pp. 115-156. 
Thibault, A., (2008b) « Les régionalismes dans La Rue Cases-Nègres (1950) de Joseph Zobel », in Thibault, A. (coord.), Richesses du français et géographie linguistique. Bruxelles, De Boeck / Duculot, vol. 2, pp. 227-314.

Thibault, A., (2010) «L'œuvre d'Aimé Césaire et le français régional antillais », in Cheymol, M. \& Ph. Ollé-Laprune (éds.), Aimé Césaire à l'œuvre. Paris, Éditions des Archives Contemporaines, pp. 43-86.

Thibault, A., (2014) «L'antillanité est-elle traduisible ? Analyse des diatopismes de Éloge de la créolité dans sa traduction anglaise », in Farina, A. \& V. Zotti (dir.), La variation lexicale des français. Dictionnaires, bases de données, corpus. Hommage à Claude Poirier. Paris, Honoré Champion, pp. 263-292.

Thibault, A. \& P. Drouin, (2010) «Le lexique de Joseph Zobel, auteur antillais : extraction semi-automatique des particularismes lexicaux » in Iliescu, M., Siller-Runggaldier, H. \& P. Danler (éds.), Actes du XXVe Congrès International de Linguistique et de Philologie Romanes, Innsbruck, 3-8 septembre 2007. Berlin / New York, De Gruyter, t. 7, pp. 291-300.

$\mathrm{TLF}=$ Trésor de la langue française. Dictionnaire de la langue du XIX et du XXe siècle (1789-1960). Paris, CNRS, 1971-1994. [En ligne]. Version informatisée disponible sur : http://atilf.atilf.fr/tlf.htm [Dernier accès le 20/02/2017].

Tirolien, G., (1961) Balles d'or. Paris, Éditions Présence Africaine.

Valdman, A. et al., (2007) Haitian Creole-English Bilingual Dictionary. Bloomington, Indiana University.

Venuti, L., (1995) The translator invisibility: a history of translation. London, Routledge.

Venuti, L., (1998) «Strategies of translation », in Baker, M. (éd.), Encyclopedia of Translation Studies. London / New York, Routledge, pp. 240-244.

Vidal Claramonte, M. C. A., (2007) « Después del giro cultural de la traducción », in Ortega Arjonilla, E. (éd.), El giro cultural de la traducción. Reflexiones teóricas y aplicaciones didácticas. Frankfurt am Main, Peter Lang, pp. 61-68.

Wecksteen, C. \& A. El Kaladi, (éds.) (2007) La traductologie dans tous ses états. Arras, Artois Presses Université.

Zanoaga, F., (2012) Contribution à la description des particularités lexicales du français régional des Antilles. Étude d'un corpus de littérature contemporaine : les romans L'Homme-au-Bâton (1992) et L'Envers du décor (2006) de l'auteur antillais Ernest Pépin. Thèse de doctorat. Paris, Université de Paris IV-Sorbonne.

Zobel, J., (2002) Le soleil m'a dit.... Matoury, Ibis Rouge Éditions. 